NOTICE: This is the author's version of a work that was accepted for publication in Systems and Control Letters. Changes resulting from the publishing process, such as peer review, editing, corrections, structural formatting, and other quality control mechanisms may not be reflected in this document. Changes may have been made to this work since it was submitted for publication. A definitive version was subsequently published in Systems and Control Letters, Vol. 72, (2014). doi: 10.1016/j.sysconle.2014.07.001 


\title{
A computational method for solving time-delay optimal control problems with free terminal time
}

\author{
Chongyang Liu ${ }^{\mathrm{a}}$, Ryan Loxton ${ }^{\mathrm{b}, \mathrm{c}}$, Kok Lay Teo ${ }^{\mathrm{b}}$ \\ ${ }^{a}$ School of Mathematics and Information Science, Shandong Institute of Business and Technology, Yantai, China \\ ${ }^{b}$ Department of Mathematics and Statistics, Curtin University, Perth, Australia \\ ${ }^{c}$ Institute of Cyber-Systems and Control, Zhejiang University, Hangzhou, China
}

\begin{abstract}
This paper considers a class of optimal control problems for general nonlinear time-delay systems with free terminal time. We first show that for this class of problems, the well-known time-scaling transformation for mapping the free time horizon into a fixed time interval yields a new time-delay system in which the time-delays are variable. Then, we introduce a control parameterization scheme to approximate the control variables in the new system by piecewise-constant functions. This yields an approximate finite-dimensional optimization problem with three types of decision variables: the control heights, the control switching times, and the terminal time in the original system (which influences the variable time-delays in the new system). We develop a gradient-based optimization approach for solving this approximate problem. Simulation results are also provided to demonstrate the effectiveness of the proposed approach.
\end{abstract}

Key words: Time-delay system; Optimal control; Free terminal time; Control parameterization; Nonlinear optimization

\section{Introduction}

The terminal time in an optimal control problem can be either fixed or variable. For optimal control problems with variable terminal time, there are two main categories: problems in which the terminal time is a free decision parameter [1,2]; and problems in which the terminal time varies according to some stopping criterion $[3,4,5]$. This paper is concerned with problems in the first category.

For such problems, a well-known time-scaling transformation is available for mapping the free terminal time into a fixed time point $[3,6]$. This transformation is very useful because it converts an optimal control problem with free terminal time into a standard optimal control problem with fixed terminal time, which can (in principle) be solved using conventional methods. However, when applied to time-delay optimal control problems, the time-scaling transformation results in unexpected difficulties. In particular, as we will show in this

\footnotetext{
Email address: r.loxton@curtin.edu.au (Ryan Loxton)

Preprint submitted to Systems \& Control Letters
}

paper, applying the time-scaling transformation to a time-delay system yields a new system in which the time-delays are variable and actually depend on the terminal time. Consequently, time-delay optimal control problems with free terminal time are a major computational challenge.

We are only aware of one reference (reference [7]) that tackles this class of problems. This reference describes a two-stage optimization approach in which the terminal time is optimized in the outer stage, and the control function is optimized in the inner stage. The advantage of this approach is that the inner stage only requires solving optimal control problems with fixed terminal time - a task that can be readily implemented using existing numerical techniques such as control parameterization [8] or state discretization [9]. However, this approach also has two disadvantages: (i) a sequence of optimal control problems must be solved, not just one; and (ii) the time-delays in the governing dynamic system must be commensurate with each other.

The purpose of this paper is to develop an alternative method that does not suffer from these limitations. Our approach involves applying the conJuly 24, 2014 
trol parameterization method, in which the control is approximated by a piecewise-constant function, to the equivalent problem obtained via the timescaling transformation. This yields an approximate finite-dimensional optimization problem, whose decision variables are the heights for the approximate control, the switching times for the approximate control, and the terminal time in the original system (which influences the variable time-delays in the transformed system). The main contribution of this paper is an algorithm for computing the gradients of the cost and constraint functionals with respect to these decision variables. By exploiting this algorithm, the approximate problem can be solved efficiently using gradient-based optimization techniques. We conclude the paper by validating this approach on a numerical example involving the harvesting of a renewable resource.

\section{Problem formulation}

\subsection{Optimal control problem}

Consider the following nonlinear control system with $m$ time-delays:

$$
\begin{aligned}
\dot{x}(t) & =f\left(t, x\left(t-\alpha_{0}\right), \ldots, x\left(t-\alpha_{m}\right), u(t)\right), \\
t \in[0, T], & \\
x(t) & =\phi(t), \quad t \leq 0,
\end{aligned}
$$

where $x(t) \in R^{n}$ is the state vector; $u(t) \in R^{r}$ is the control vector; $T>0$ is a free terminal time; $\alpha_{0}=0$ and $\alpha_{i}>0, i=1, \ldots, m$, are given time-delays; and $f: R \times R^{(m+1) n} \times R^{r} \rightarrow R^{n}$ and $\phi: R \rightarrow R^{n}$ are given functions.

The terminal time $T$ in system (1a) is a free decision variable. Define

$$
\mathcal{T}:=\left\{\gamma \in R: T_{\min } \leq \gamma \leq T_{\max }\right\}
$$

where $T_{\min }$ and $T_{\max }$ are the lower and upper bounds for the terminal time, respectively. Any $T \in \mathcal{T}$ is called an admissible terminal time.

Furthermore, define

$$
U:=\left\{v \in R^{r}: a_{j} \leq v_{j} \leq b_{j}, j=1, \ldots, r\right\},
$$

where $a_{j}$ and $b_{j}, j=1, \ldots, r$, are the lower and upper bounds for the $j$ th control variable, respectively. Any measurable function $u:[0, \infty) \rightarrow R^{r}$ such that $u(t) \in U$ for almost all $t \geq 0$ is called an admissible control. Let $\mathcal{U}$ denote the class of all such admissible controls. Accordingly, any pair $(T, u) \in \mathcal{T} \times \mathcal{U}$ is called an admissible pair for system (1).
We assume throughout this paper that the following conditions are satisfied.

Assumption 1. The functions $f$ and $\phi$ are continuously differentiable.

Assumption 2. There exists a real number $L>$ 0 such that for all $\eta \in\left[0, T_{\max }\right], y^{i} \in R^{n}, i=$ $0, \ldots, m$, and $v \in U$,

$$
\left|f\left(\eta, y^{0}, \ldots, y^{m}, v\right)\right| \leq L\left(1+\left|y^{0}\right|+\cdots+\left|y^{m}\right|\right),
$$

where $|\cdot|$ denotes the Euclidean norm.

Assumptions 1 and 2 ensure that system (1) has a unique solution $x(\cdot \mid u)$ corresponding to each $u \in \mathcal{U}$ [10]. This solution is called the state trajectory.

We suppose that system (1) is subject to the following canonical constraints:

$$
g_{k}(T, u)=\Phi_{k}(T, x(T \mid u)) \begin{cases}=0, & k \in \mathcal{E}, \\ \geq 0, & k \in \mathcal{I},\end{cases}
$$

where $\mathcal{E}$ is the index set for the equality constraints; $\mathcal{I}$ is the index set for the inequality constraints; and $\Phi_{k}: R \times R^{n} \rightarrow R, k \in \mathcal{E} \cup \mathcal{I}$, are given continuously differentiable functions. Note that we can easily transform integral constraints into the form of (4) by introducing additional state variables. For example, consider the following integral term:

$$
\int_{0}^{T} \mathcal{L}\left(t, x\left(t-\alpha_{0}\right), \ldots, x\left(t-\alpha_{m}\right), u(t)\right) d t,
$$

where $\mathcal{L}: R \times R^{(m+1) n} \times R^{r} \rightarrow R$ is a given continuously differentiable function. Clearly, this term can be replaced by $x_{n+1}(T)$, where $x_{n+1}$ is a new state variable satisfying the dynamics

$$
\begin{aligned}
& \dot{x}_{n+1}(t)=\mathcal{L}\left(t, x\left(t-\alpha_{0}\right), \ldots, x\left(t-\alpha_{m}\right), u(t)\right), \\
& t \in[0, T], \\
& x_{n+1}(t)=0, t \leq 0 .
\end{aligned}
$$

Thus, there is no loss of generality in ignoring integral terms in the constraint functions (4).

We now state our optimal control problem as follows.

Problem (P). Find an admissible pair $(T, u) \in$ $\mathcal{T} \times \mathcal{U}$ such that the cost functional

$$
g_{0}(T, u)=\Phi_{0}(T, x(T \mid u))
$$

is minimized subject to the canonical constraints (4), where $\Phi_{0}: R \times R^{n} \rightarrow R$ is a given continuously differentiable function. 


\subsection{Problem transformation}

Problem (P) is difficult to solve numerically because the time-delay system (1) must be integrated over a variable time horizon. For non-delay systems, this difficulty can be overcome by applying the following time-scaling transformation to map the variable interval $[0, T]$ into the fixed interval $[0,1]$ :

$$
t=t(s)=T s,
$$

where $s \in[0,1]$ is a new time variable. Clearly, $s=0$ corresponds to $t=0$, and $s=1$ corresponds to $t=T$. This transformation is well-known in the optimal control of non-delay systems. We now investigate its use for the time-delay system (1). Let $\tilde{x}(s)=x(t(s))$ and $\tilde{u}(s)=u(t(s))$. Then

$$
\begin{aligned}
& \dot{\tilde{x}}(s)=\frac{d}{d s}\{x(t(s))\}=\frac{d x(t(s))}{d t} \frac{d t(s)}{d s} \\
& =T f\left(T s, x\left(T s-\alpha_{0}\right), \ldots, x\left(T s-\alpha_{m}\right), u(T s)\right) \\
& =T f\left(T s, \tilde{x}\left(s-\alpha_{0} T^{-1}\right), \ldots, \tilde{x}\left(s-\alpha_{m} T^{-1}\right), \tilde{u}(s)\right) .
\end{aligned}
$$

The initial condition (1b) becomes

$$
\tilde{x}(s)=\phi(T s), \quad s \leq 0 .
$$

Thus, the original control system (1) can be transformed into the following form:

$$
\begin{aligned}
& \dot{\tilde{x}}(s)= T f\left(T s, \tilde{x}\left(s-\alpha_{0} T^{-1}\right),\right. \\
&\left.\quad \ldots, \tilde{x}\left(s-\alpha_{m} T^{-1}\right), \tilde{u}(s)\right), s \in[0,1], \\
& \tilde{x}(s)=\phi(T s), \quad s \leq 0 .
\end{aligned}
$$

Let $\tilde{\mathcal{U}}$ be the class of all measurable functions $\tilde{u}:[0,1] \rightarrow R^{r}$ such that $\tilde{u}(s) \in U$ for almost all $s \in[0,1]$. Any pair $(T, \tilde{u}) \in \mathcal{T} \times \tilde{\mathcal{U}}$ is called an admissible pair. Let $\tilde{x}(\cdot \mid T, \tilde{u})$ denote the solution of system (7) corresponding to a given admissible pair $(T, \tilde{u}) \in \mathcal{T} \times \tilde{\mathcal{U}}$. Then the canonical constraints (4) become

$$
\tilde{g}_{k}(T, \tilde{u})=\Phi_{k}(T, \tilde{x}(1 \mid T, \tilde{u})) \begin{cases}=0, & k \in \mathcal{E}, \\ \geq 0, & k \in \mathcal{I} .\end{cases}
$$

Thus, Problem $(\mathrm{P})$ is equivalent to the following optimal control problem with fixed terminal time.

Problem (EP). Find an admissible pair $(T, \tilde{u}) \in$ $\mathcal{T} \times \tilde{\mathcal{U}}$ such that the cost functional

$$
\tilde{g}_{0}(T, \tilde{u})=\Phi_{0}(T, \tilde{x}(1 \mid T, \tilde{u}))
$$

is minimized subject to the canonical constraints (8).
Note that the time-delays in system (7a) depend on the decision parameter $T$. Thus, when applied to time-delay systems, the time-scaling transformation (6) yields a non-standard optimal control problem with variable time-delays. This is quite different from the situation for non-delay systems, in which the time-scaling transformation (6) yields a standard optimal control problem that can be solved using conventional techniques. Nevertheless, despite the complications caused by the variable time-delays, we will show in the next section that Problem (EP) can be solved using a combination of the control parameterization technique and gradient-based optimization methods.

\section{Numerical algorithm}

\subsection{Control parameterization}

To solve Problem (EP), we apply the control parameterization scheme [8]. This involves approximating the control by a piecewise-constant function with a finite number of discontinuities.

For a given integer $q \geq 1$, let $\tau_{0}=0$ and $\tau_{q+1}=1$. Within the interval $[0,1]$, we allow the approximate piecewise-constant control to switch value at the intermediate time points $\tau_{l}, l=1, \ldots, q$. These switching times satisfy the constraints

$$
\tau_{l}-\tau_{l-1} \geq \Delta, \quad l=1, \ldots, q+1,
$$

where $\Delta>0$ is the minimum switching duration. Thus, the approximate control can be expressed as

$$
\tilde{u}^{q}(s)=\sum_{l=1}^{q+1} \sigma^{l} \chi_{\left[\tau_{l-1}, \tau_{l}\right)}(s),
$$

where, for a given interval $I, \chi_{I}: R \rightarrow R$ denotes the indicator function of $I$ defined by

$$
\chi_{I}(s)= \begin{cases}1, & \text { if } s \in I \\ 0, & \text { otherwise }\end{cases}
$$

For $\tilde{u}^{q}$ to be an admissible control, we must have $\sigma^{l}=\left(\sigma_{1}^{l}, \ldots, \sigma_{r}^{l}\right)^{\top} \in U$ for each $l=1, \ldots, q+1$. Let $\Xi$ denote the set of all $\sigma=\left(\left(\sigma^{1}\right)^{\top}, \ldots,\left(\sigma^{q+1}\right)^{\top}\right)^{\top} \in$ $R^{(q+1) r}$ satisfying this requirement. Furthermore, let $\Gamma$ denote the set of all $\tau=\left(\tau_{1}, \ldots, \tau_{q}\right)^{\top} \in R^{q}$ satisfying constraint (10). Clearly, for each $(\sigma, \tau) \in$ $\Xi \times \Gamma$, the corresponding piecewise-constant control $\tilde{u}^{q}$ is admissible for Problem (EP), i.e., $\tilde{u}^{q} \in \tilde{\mathcal{U}}$. Accordingly, any triple $(T, \sigma, \tau) \in \mathcal{T} \times \Xi \times \Gamma$ is called an admissible triple. 
After applying the control approximation (11), the dynamic system (7) becomes

$$
\begin{aligned}
& \dot{\tilde{x}}(s)=T f\left(T s, \tilde{x}\left(s-\alpha_{0} T^{-1}\right), \ldots, \tilde{x}\left(s-\alpha_{m} T^{-1}\right), \sigma^{l}\right), \\
& \quad s \in\left[\tau_{l-1}, \tau_{l}\right), \quad l=1, \ldots, q+1, \quad(13 \mathrm{a}) \\
& \tilde{x}(s)=\phi(T s), \quad s \leq 0 .
\end{aligned}
$$

Let $\tilde{x}^{q}(\cdot \mid T, \sigma, \tau)$ denote the solution of system (13). Then, the canonical constraints (8) become

$$
\begin{aligned}
& \tilde{g}_{k}^{q}(T, \sigma, \tau) \\
& =\Phi_{k}\left(T, \tilde{x}^{q}(1 \mid T, \sigma, \tau)\right)\left\{\begin{array}{l}
=0, k \in \mathcal{E}, \\
\geq 0, k \in \mathcal{I} .
\end{array}\right.
\end{aligned}
$$

On this basis, we obtain the following finitedimensional approximate problem.

Problem $(\operatorname{EP}(q))$. Find an admissible triple $(T, \sigma, \tau) \in \mathcal{T} \times \Xi \times \Gamma$ such that the cost functional

$$
\tilde{g}_{0}^{q}(T, \sigma, \tau)=\Phi_{0}\left(T, \tilde{x}^{q}(1 \mid T, \sigma, \tau)\right)
$$

is minimized subject to the canonical constraints (14).

Problem $(\mathrm{EP}(q))$ is a finite-dimensional optimization problem whose decision variables are the heights of the approximate control, the switching times of the approximate control and the terminal time in the original system. It is well known that variable switching times pose a significant challenge for conventional numerical optimization techniques $[11,12,13]$. To overcome this challenge, an extended version of the time-scaling transformation (6) is typically used to map the variable switching times to fixed time points in a new time horizon [11]. However, as discussed in [8], this extended time-scaling transformation is not applicable to time-delay systems. Indeed, the transformation fails even for time-delay systems with fixed time-delays, let alone time-delay systems with variable time-delays such as system (13). Thus, a new approach is needed to solve Problem $(\operatorname{EP}(q))$.

\subsection{Gradient computation}

Problem (EP) can be approximated by a sequence of finite-dimensional optimization problems in the form of Problem $(\operatorname{EP}(q))$. In essence, each Problem $(\operatorname{EP}(q))$ is a constrained mathematical programming problem that can be solved using gradient-based optimization techniques [14]. However, to do this, the gradients of the cost and constraint functionals are required.
Note that the cost functional (15) is in a similar form to the canonical constraints (14). Hence, the gradients of the cost and constraint functionals can be computed in a unified fashion. Define

$$
\begin{aligned}
& \hat{f}^{l}(s \mid T, \sigma, \tau) \\
& =T f\left(T s, \tilde{x}^{q}\left(s-\alpha_{0} T^{-1}\right), \ldots, \tilde{x}^{q}\left(s-\alpha_{m} T^{-1}\right), \sigma^{l}\right),
\end{aligned}
$$

where $\tilde{x}^{q}(\cdot)=\tilde{x}^{q}(\cdot \mid T, \sigma, \tau)$. Furthermore, define

$$
\psi(s \mid T, \sigma, \tau)= \begin{cases}T \dot{\phi}(T s), & \text { if } s<0, \\ \hat{f}^{l}(s \mid T, \sigma, \tau), & \text { if } s \in\left[\tau_{l-1}, \tau_{l}\right) .\end{cases}
$$

In the following, we use the notation $\partial x^{i}$ to denote differentiation with respect to the $i$ th delayed state in $f$. Accordingly, let

$$
\begin{aligned}
& \frac{\partial \hat{f}^{l}(s \mid T, \sigma, \tau)}{\partial t} \\
& =T \frac{\partial f\left(T s, \tilde{x}^{q}\left(s-\alpha_{0} T^{-1}\right), \ldots, \tilde{x}^{q}\left(s-\alpha_{m} T^{-1}\right), \sigma^{l}\right)}{\partial t},
\end{aligned}
$$

$\frac{\partial \hat{f}^{l}(s \mid T, \sigma, \tau)}{\partial x^{i}}$

$=T \frac{\partial f\left(T s, \tilde{x}^{q}\left(s-\alpha_{0} T^{-1}\right), \ldots, \tilde{x}^{q}\left(s-\alpha_{m} T^{-1}\right), \sigma^{l}\right)}{\partial x^{i}}$,

$\frac{\partial \hat{f}^{l}(s \mid T, \sigma, \tau)}{\partial u}$

$=T \frac{\partial f\left(T s, \tilde{x}^{q}\left(s-\alpha_{0} T^{-1}\right), \ldots, \tilde{x}^{q}\left(s-\alpha_{m} T^{-1}\right), \sigma^{l}\right)}{\partial u}$,

where $\tilde{x}^{q}(\cdot)=\tilde{x}^{q}(\cdot \mid T, \sigma, \tau)$. Furthermore, define

$$
\mathcal{S}_{l}^{i}(T, \tau)=\left[\tau_{l-1}-\alpha_{i} T^{-1}, \tau_{l}-\alpha_{i} T^{-1}\right) .
$$

For each $k \in\{0\} \cup \mathcal{E} \cup \mathcal{I}$, consider the following costate system:

$$
\begin{aligned}
\dot{\lambda}_{k}(s) & =-\sum_{l=1}^{q+1} \sum_{i=0}^{m}\left(\frac{\partial \hat{f}^{l}\left(s+\alpha_{i} T^{-1} \mid T, \sigma, \tau\right)}{\partial x^{i}}\right)^{\top} \\
& \times \lambda_{k}\left(s+\alpha_{i} T^{-1}\right) \chi_{\mathcal{S}_{l}^{i}(T, \tau)}(s), s \in[0,1),
\end{aligned}
$$

with the terminal conditions

$$
\begin{aligned}
& \lambda_{k}(1)=\left(\frac{\partial \Phi_{k}\left(T, \tilde{x}^{q}(1 \mid T, \sigma, \tau)\right)}{\partial x}\right)^{\top}, \\
& \lambda_{k}(s)=0, \quad s>1,
\end{aligned}
$$

where $\chi_{\mathcal{S}_{l}^{i}(T, \tau)}$ is as defined in (12). Note that, if $s>1-\alpha_{i} T^{-1}$, then $s+\alpha_{i} T^{-1}>1$, and 
thus the value of $\partial \hat{f}^{l}\left(s+\alpha_{i} T^{-1}\right) / \partial x^{i}$ is undefined. However, this value has no effect on the costate dynamics (16) because $\lambda_{k}\left(s+\alpha_{i} T^{-1}\right)=0$ when $s>1-\alpha_{i} T^{-1}$ (see equation (18)).

Let $\lambda_{k}^{q}(\cdot \mid T, \sigma, \tau)$ denote the solution of the costate system (16)-(18) corresponding to the given triple $(T, \sigma, \tau) \in \mathcal{T} \times \Xi \times \Gamma$. We now express the gradients of (14) and (15) with respect to the terminal time $T$ in terms of $\lambda_{k}^{q}(\cdot \mid T, \sigma, \tau)$.

Theorem 1. Let $k \in\{0\} \cup \mathcal{E} \cup \mathcal{I}$. Then

$$
\begin{aligned}
\frac{\partial \tilde{g}_{k}^{q}(T, \sigma, \tau)}{\partial T} & =\frac{\partial \Phi_{k}\left(T, \tilde{x}^{q}(1)\right)}{\partial T}+\sum_{l=1}^{q+1} \int_{\tau_{l-1}}^{\tau_{l}} \overline{\mathcal{L}}_{l}(s) d s \\
& +\sum_{l=1}^{q+1} \sum_{i=1}^{m} \int_{\tau_{l-1}-\alpha_{i} T^{-1}}^{\tau_{l}-\alpha_{i} T^{-1}} \hat{\mathcal{L}}_{l}^{i}(s) d s, \quad(19)
\end{aligned}
$$

where $\tilde{x}^{q}(\cdot)=\tilde{x}^{q}(\cdot \mid T, \sigma, \tau)$,

$$
\begin{aligned}
& \overline{\mathcal{L}}_{l}(s)=\lambda_{k}^{q}(s \mid T, \sigma, \tau)^{\top}\left(s \frac{\partial \hat{f}^{l}(s \mid T, \sigma, \tau)}{\partial t}\right. \\
& +\sum_{i=1}^{m} \frac{\alpha_{i}}{T^{2}} \frac{\partial \hat{f}^{l}(s \mid T, \sigma, \tau)}{\partial x^{i}} \psi\left(s-\alpha_{i} T^{-1} \mid T, \sigma, \tau\right) \\
& \left.+T^{-1} \hat{f}^{l}(s \mid T, \sigma, \tau)\right)
\end{aligned}
$$

and

$$
\begin{aligned}
& \hat{\mathcal{L}}_{l}^{i}(s)=\lambda_{k}^{q}\left(s+\alpha_{i} T^{-1} \mid T, \sigma, \tau\right)^{\top} \\
& \times s \frac{\partial \hat{f}^{l}\left(s+\alpha_{i} T^{-1} \mid T, \sigma, \tau\right)}{\partial x^{i}} \dot{\phi}(T s) \chi_{(-\infty, 0)}(s) .
\end{aligned}
$$

Proof. Let $w:[0, \infty) \rightarrow R^{n}$ be an arbitrary function that is continuous and differentiable almost everywhere. Then we may express $\tilde{g}_{k}^{q}$ as follows:

$$
\begin{aligned}
& \tilde{g}_{k}^{q}(T, \sigma, \tau) \\
& =\Phi_{k}\left(T, \tilde{x}^{q}(1)\right)+\sum_{l=1}^{q+1} \int_{\tau_{l-1}}^{\tau_{l}} w(s)^{\top}\left(\hat{f}^{l}(s)-\dot{\tilde{x}}^{q}(s)\right) d s \\
& =\Phi_{k}\left(T, \tilde{x}^{q}(1)\right)+\sum_{l=1}^{q+1} \int_{\tau_{l-1}}^{\tau_{l}} w(s)^{\top} \hat{f}^{l}(s) d s \\
& \quad-\int_{0}^{1} w(s)^{\top} \dot{\tilde{x}}^{q}(s) d s,
\end{aligned}
$$

where we have omitted the arguments $T, \sigma$ and $\tau$ in $\tilde{x}^{q}(\cdot \mid T, \sigma, \tau)$ and $\hat{f}^{l}(\cdot \mid T, \sigma, \tau)$ for simplicity.
Applying integration by parts to the last integral term gives

$$
\begin{gathered}
\tilde{g}_{k}^{q}(T, \sigma, \tau)=\Phi_{k}\left(T, \tilde{x}^{q}(1)\right)-w(1)^{\top} \tilde{x}^{q}(1)+w(0)^{\top} \phi(0) \\
+\int_{0}^{1} \dot{w}(s)^{\top} \tilde{x}^{q}(s) d s+\sum_{l=1}^{q+1} \int_{\tau_{l-1}}^{\tau_{l}} w(s)^{\top} \hat{f}^{l}(s) d s .
\end{gathered}
$$

Differentiating (22) with respect to $T$ gives

$$
\begin{aligned}
& \frac{\partial \tilde{g}_{k}^{q}(T, \sigma, \tau)}{\partial T}=\left(\frac{\partial \Phi_{k}\left(T, \tilde{x}^{q}(1)\right)}{\partial x}-w(1)^{\top}\right) \frac{\partial \tilde{x}^{q}(1)}{\partial T} \\
& +\frac{\partial \Phi_{k}\left(T, \tilde{x}^{q}(1)\right)}{\partial T}+\int_{0}^{1} \dot{w}(s)^{\top} \frac{\partial \tilde{x}^{q}(s)}{\partial T} d s \\
& +\sum_{l=1}^{q+1} \int_{\tau_{l-1}}^{\tau_{l}} w(s)^{\top}\left\{s \frac{\partial \hat{f}^{l}(s)}{\partial t}+\sum_{i=1}^{m}\left(\frac{\alpha_{i}}{T^{2}} \frac{\partial \hat{f}^{l}(s)}{\partial x^{i}}\right.\right. \\
& \left.\left.\quad \times \psi\left(s-\alpha_{i} T^{-1}\right)\right)+T^{-1} \hat{f}^{l}(s)\right\} d s \\
& \quad+\sum_{l=1}^{q+1} \sum_{i=0}^{m} \int_{\tau_{l-1}}^{\tau_{l}} w(s)^{\top} \frac{\partial \hat{f}^{l}(s)}{\partial x^{i}} \frac{\partial \tilde{x}^{q}\left(s-\alpha_{i} T^{-1}\right)}{\partial T} d s
\end{aligned}
$$

where we have omitted the arguments $T, \sigma$ and $\tau$ in $\psi\left(s-\alpha_{i} T^{-1} \mid T, \sigma, \tau\right)$. Performing a change of variable in the last term on the right-hand side of (23) yields

$$
\begin{aligned}
& \sum_{l=1}^{q+1} \sum_{i=0}^{m} \int_{\tau_{l-1}}^{\tau_{l}} w(s)^{\top} \frac{\partial \hat{f}^{l}(s)}{\partial x^{i}} \frac{\partial \tilde{x}^{q}\left(s-\alpha_{i} T^{-1}\right)}{\partial T} d s \\
& =\sum_{l=1}^{q+1} \sum_{i=0}^{m} \int_{\tau_{l-1}-\alpha_{i} T^{-1}}^{\tau_{l}-\alpha_{i} T^{-1}}\left(w\left(s+\alpha_{i} T^{-1}\right)^{\top}\right. \\
& \left.\quad \times \frac{\partial \hat{f}^{l}\left(s+\alpha_{i} T^{-1}\right)}{\partial x^{i}} \frac{\partial \tilde{x}^{q}(s)}{\partial T}\right) d s .
\end{aligned}
$$

Since $\tilde{x}^{q}(s)=\phi(T s)$ for all $s \leq 0$, equation (24) can 
be rewritten as

$$
\begin{aligned}
& \sum_{l=1}^{q+1} \sum_{i=0}^{m} \int_{\tau_{l-1}}^{\tau_{l}} w(s)^{\top} \frac{\partial \hat{f}^{l}(s)}{\partial x^{i}} \frac{\partial \tilde{x}^{q}\left(s-\alpha_{i} T^{-1}\right)}{\partial T} d s \\
& =\sum_{l=1}^{q+1} \sum_{i=0}^{m} \int_{\tau_{l-1}-\alpha_{i} T^{-1}}^{\tau_{l}-\alpha_{i} T^{-1}}\left(w\left(s+\alpha_{i} T^{-1}\right)^{\top}\right. \\
& \left.\quad \times \frac{\partial \hat{f}^{l}\left(s+\alpha_{i} T^{-1}\right)}{\partial x^{i}} \frac{\partial \tilde{x}^{q}(s)}{\partial T} \chi_{[0,+\infty)}(s)\right) d s \\
& \quad+\sum_{l=1}^{q+1} \sum_{i=1}^{m} \int_{\tau_{l-1}-\alpha_{i} T^{-1}}^{\tau_{l}-\alpha_{i} T^{-1}}\left(w\left(s+\alpha_{i} T^{-1}\right)^{\top}\right. \\
& \left.\quad \times s \frac{\partial \hat{f}^{l}\left(s+\alpha_{i} T^{-1}\right)}{\partial x^{i}} \dot{\phi}(T s) \chi_{(-\infty, 0)}(s)\right) d s
\end{aligned}
$$

where $\chi_{[0,+\infty)}$ and $\chi_{(-\infty, 0)}$ are as defined in (12). Choosing $w(\cdot)=\lambda_{k}^{q}(\cdot \mid T, \sigma, \tau)$ and substituting (25) into (23) yields

$$
\begin{aligned}
& \frac{\partial \tilde{g}_{k}^{q}(T, \sigma, \tau)}{\partial T}=\left(\frac{\partial \Phi_{k}\left(T, \tilde{x}^{q}(1)\right)}{\partial x}-\lambda_{k}^{q}(1)^{\top}\right) \frac{\partial \tilde{x}^{q}(1)}{\partial T} \\
& +\frac{\partial \Phi_{k}\left(T, \tilde{x}^{q}(1)\right)}{\partial T}+\sum_{l=1}^{q+1} \int_{\tau_{l-1}}^{\tau_{l}} \overline{\mathcal{L}}_{l}(s) d s \\
& +\int_{0}^{1}\left\{\dot{\lambda}_{k}^{q}(s)^{\top}+\sum_{l=1}^{q+1} \sum_{i=0}^{m}\left(\lambda_{k}^{q}\left(s+\alpha_{i} T^{-1}\right)^{\top}\right.\right. \\
& \left.\left.\quad \times \frac{\partial \hat{f}^{l}\left(s+\alpha_{i} T^{-1}\right)}{\partial x^{i}} \chi_{\mathcal{S}_{l}^{i}(T, \tau)}(s)\right)\right\} \frac{\partial \tilde{x}^{q}(s)}{\partial T} d s \\
& +\sum_{l=1}^{q+1} \sum_{i=1}^{m} \int_{\tau_{l-1}-\alpha_{i} T^{-1}}^{\tau_{l}-\alpha_{i} T^{-1}} \hat{\mathcal{L}}_{l}^{i}(s) d s,
\end{aligned}
$$

where we have omitted the arguments $T, \sigma$ and $\tau$ in $\lambda_{k}^{q}(\cdot \mid T, \sigma, \tau)$, and $\overline{\mathcal{L}}_{l}$ and $\hat{\mathcal{L}}_{l}^{i}$ are as defined in (20) and (21), respectively. Substituting (16)-(18) into (26) completes the proof.

The next theorem gives the gradients of (14) and (15) with respect to $\sigma$.

Theorem 2. Let $k \in\{0\} \cup \mathcal{E} \cup \mathcal{I}$ and $l \in\{1, \ldots, q+$ 1\}. Then

$\frac{\partial \tilde{g}_{k}^{q}(T, \sigma, \tau)}{\partial \sigma^{l}}=\int_{\tau_{l-1}}^{\tau_{l}} \lambda_{k}^{q}(s \mid T, \sigma, \tau)^{\top} \frac{\partial \hat{f}^{l}(s \mid T, \sigma, \tau)}{\partial u} d s$.

Proof. Let $w(\cdot)$ be as defined in the proof of Theorem 1. Recall from (22) that

$$
\begin{gathered}
\tilde{g}_{k}^{q}(T, \sigma, \tau)=\Phi_{k}\left(T, \tilde{x}^{q}(1)\right)-w(1)^{\top} \tilde{x}^{q}(1)+w(0)^{\top} \phi(0) \\
+\int_{0}^{1} \dot{w}(s)^{\top} \tilde{x}^{q}(s) d s+\sum_{\rho=1}^{q+1} \int_{\tau_{\rho-1}}^{\tau_{\rho}} w(s)^{\top} \hat{f}^{\rho}(s) d s,
\end{gathered}
$$

where, as in the proof of Theorem 1, we have omitted the arguments $T, \sigma$ and $\tau$ for clarity.

Differentiating this equation with respect to $\sigma^{l}$ gives

$$
\begin{gathered}
\frac{\partial \tilde{g}_{k}^{q}(T, \sigma, \tau)}{\partial \sigma^{l}}=\left(\frac{\partial \Phi_{k}\left(T, \tilde{x}^{q}(1)\right)}{\partial x}-w(1)^{\top}\right) \frac{\partial \tilde{x}^{q}(1)}{\partial \sigma^{l}} \\
+\int_{0}^{1} \dot{w}(s)^{\top} \frac{\partial \tilde{x}^{q}(s)}{\partial \sigma^{l}} d s+\int_{\tau_{l-1}}^{\tau_{l}} w(s)^{\top} \frac{\partial \hat{f}^{l}(s)}{\partial u} d s \\
+\sum_{\rho=1}^{q+1} \sum_{i=0}^{m} \int_{\tau_{\rho-1}}^{\tau_{\rho}} w(s)^{\top} \frac{\partial \hat{f}^{\rho}(s)}{\partial x^{i}} \frac{\partial \tilde{x}^{q}\left(s-\alpha_{i} T^{-1}\right)}{\partial \sigma^{l}} d s .
\end{gathered}
$$

Performing a change of variable in the last term on the right-hand side of (27) yields

$$
\begin{aligned}
& \sum_{\rho=1}^{q+1} \sum_{i=0}^{m} \int_{\tau_{\rho-1}}^{\tau_{\rho}} w(s)^{\top} \frac{\partial \hat{f}^{\rho}(s)}{\partial x^{i}} \frac{\partial \tilde{x}^{q}\left(s-\alpha_{i} T^{-1}\right)}{\partial \sigma^{l}} d s \\
& =\sum_{\rho=1}^{q+1} \sum_{i=0}^{m} \int_{\tau_{\rho-1}-\alpha_{i} T^{-1}}^{\tau_{\rho}-\alpha_{i} T^{-1}}\left(w\left(s+\alpha_{i} T^{-1}\right)^{\top}\right. \\
& \left.\quad \times \frac{\partial \hat{f}^{\rho}\left(s+\alpha_{i} T^{-1}\right)}{\partial x^{i}} \frac{\partial \tilde{x}^{q}(s)}{\partial \sigma^{l}}\right) d s .
\end{aligned}
$$

Since $\tilde{x}^{q}(s)=\phi(T s)$ for all $s \leq 0$, it is clear that $\partial \tilde{x}^{q}(s) / \partial \sigma^{l}=0$ for all $s \leq 0$. Thus, (28) can be rewritten as

$$
\begin{aligned}
& \sum_{\rho=1}^{q+1} \sum_{i=0}^{m} \int_{\tau_{\rho-1}}^{\tau_{\rho}} w(s)^{\top} \frac{\partial \hat{f}^{\rho}(s)}{\partial x^{i}} \frac{\partial \tilde{x}^{q}\left(s-\alpha_{i} T^{-1}\right)}{\partial \sigma^{l}} d s \\
& =\sum_{\rho=1}^{q+1} \sum_{i=0}^{m} \int_{\tau_{\rho-1}-\alpha_{i} T^{-1}}^{\tau_{\rho}-\alpha_{i} T^{-1}}\left(w\left(s+\alpha_{i} T^{-1}\right)^{\top}\right. \\
& \left.\quad \times \frac{\partial \hat{f}^{\rho}\left(s+\alpha_{i} T^{-1}\right)}{\partial x^{i}} \frac{\partial \tilde{x}^{q}(s)}{\partial \sigma^{l}} \chi_{[0,+\infty)}(s)\right) d s,
\end{aligned}
$$

where $\chi_{[0,+\infty)}$ is as defined in (12). Substituting (29) into (27) yields

$$
\begin{aligned}
& \frac{\partial \tilde{g}_{k}^{q}(T, \sigma, \tau)}{\partial \sigma^{l}}=\left(\frac{\partial \Phi_{k}\left(T, \tilde{x}^{q}(1)\right)}{\partial x}-w(1)^{\top}\right) \frac{\partial \tilde{x}^{q}(1)}{\partial \sigma^{l}} \\
& +\int_{\tau_{l-1}}^{\tau_{l}} w(s)^{\top} \frac{\partial \hat{f}^{l}(s)}{\partial u} d s+\int_{0}^{1}\left\{\dot{w}(s)^{\top}\right. \\
& \quad+\sum_{\rho=1}^{q+1} \sum_{i=0}^{m}\left(w\left(s+\alpha_{i} T^{-1}\right)^{\top} \frac{\partial \hat{f}^{\rho}\left(s+\alpha_{i} T^{-1}\right)}{\partial x^{i}}\right. \\
& \left.\left.\quad \times \chi_{\mathcal{S}_{\rho}^{i}(T, \tau)}(s)\right)\right\} \frac{\partial \tilde{x}^{q}(s)}{\partial \sigma^{l}} d s .
\end{aligned}
$$

Choosing $w(\cdot)=\lambda_{k}^{q}(\cdot \mid T, \sigma, \tau)$ and applying (16)(18) completes the proof. 
The final theorem gives the gradients of (14) and (15) with respect to $\tau$.

Theorem 3. Let $k \in\{0\} \cup \mathcal{E} \cup \mathcal{I}$ and $l \in\{1, \ldots, q\}$. Then

$$
\begin{aligned}
& \frac{\partial \tilde{g}_{k}^{q}(T, \sigma, \tau)}{\partial \tau_{l}}=\lambda_{k}^{q}\left(\tau_{l} \mid T, \sigma, \tau\right)^{\top} \hat{f}^{l}\left(\tau_{l} \mid T, \sigma, \tau\right) \\
& -\lambda_{k}^{q}\left(\tau_{l} \mid T, \sigma, \tau\right)^{\top} \hat{f}^{l+1}\left(\tau_{l} \mid T, \sigma, \tau\right) .
\end{aligned}
$$

Proof. Let $w(\cdot)$ be as defined in the proof of Theorems 1 and 2. Recall from (22) that

$$
\begin{gathered}
\tilde{g}_{k}^{q}(T, \sigma, \tau)=\Phi_{k}\left(T, \tilde{x}^{q}(1)\right)-w(1)^{\top} \tilde{x}^{q}(1)+w(0)^{\top} \phi(0) \\
+\int_{0}^{1} \dot{w}(s)^{\top} \tilde{x}^{q}(s) d s+\sum_{\rho=1}^{q+1} \int_{\tau_{\rho-1}}^{\tau_{\rho}} w(s)^{\top} \hat{f}^{\rho}(s) d s,
\end{gathered}
$$

where, as in the proof of Theorems 1 and 2, we have omitted the arguments $T, \sigma$ and $\tau$ for clarity.

Differentiating this equation with respect to $\tau_{l}$ gives

$$
\begin{aligned}
& \frac{\partial \tilde{g}_{k}^{q}(T, \sigma, \tau)}{\partial \tau_{l}}=\left(\frac{\partial \Phi_{k}\left(T, \tilde{x}^{q}(1)\right)}{\partial x}-w(1)^{\top}\right) \frac{\partial \tilde{x}^{q}(1)}{\partial \tau_{l}} \\
& \quad+\int_{0}^{1} \dot{w}(s)^{\top} \frac{\partial \tilde{x}^{q}(s)}{\partial \tau_{l}} d s \\
& +w\left(\tau_{l}\right)^{\top} \hat{f}^{l}\left(\tau_{l}\right)-w\left(\tau_{l}\right)^{\top} \hat{f}^{l+1}\left(\tau_{l}\right) \\
& +\sum_{\rho=1}^{q+1} \sum_{i=0}^{m} \int_{\tau_{\rho-1}}^{\tau_{\rho}}\left(w(s)^{\top} \frac{\partial \hat{f}^{\rho}(s)}{\partial x^{i}} \frac{\partial \tilde{x}^{q}\left(s-\alpha_{i} T^{-1}\right)}{\partial \tau_{l}}\right) d s .
\end{aligned}
$$

Performing a change of variable in the last term on the right-hand side of (31) yields

$$
\begin{aligned}
& \sum_{\rho=1}^{q+1} \sum_{i=0}^{m} \int_{\tau_{\rho-1}}^{\tau_{\rho}} w(s)^{\top} \frac{\partial \hat{f}^{\rho}(s)}{\partial x^{i}} \frac{\partial \tilde{x}^{q}\left(s-\alpha_{i} T^{-1}\right)}{\partial \tau_{l}} d s \\
& =\sum_{\rho=1}^{q+1} \sum_{i=0}^{m} \int_{\tau_{\rho-1}-\alpha_{i} T^{-1}}^{\tau_{\rho}-\alpha_{i} T^{-1}}\left(w\left(s+\alpha_{i} T^{-1}\right)^{\top}\right. \\
& \left.\quad \times \frac{\partial \hat{f}^{\rho}\left(s+\alpha_{i} T^{-1}\right)}{\partial x^{i}} \frac{\partial \tilde{x}^{q}(s)}{\partial \tau_{l}}\right) d s .
\end{aligned}
$$

Clearly, since $\tilde{x}^{q}(s)=\phi(T s)$ for all $s \leq 0$, equation (32) can be rewritten as

$$
\begin{aligned}
& \sum_{\rho=1}^{q+1} \sum_{i=0}^{m} \int_{\tau_{\rho-1}}^{\tau_{\rho}} w(s)^{\top} \frac{\partial \hat{f}^{\rho}(s)}{\partial x^{i}} \frac{\partial \tilde{x}^{q}\left(s-\alpha_{i} T^{-1}\right)}{\partial \tau_{l}} d s \\
& =\sum_{\rho=1}^{q+1} \sum_{i=0}^{m} \int_{\tau_{\rho-1}-\alpha_{i} T^{-1}}^{\tau_{\rho}-\alpha_{i} T^{-1}}\left(w\left(s+\alpha_{i} T^{-1}\right)^{\top}\right. \\
& \left.\quad \times \frac{\partial \hat{f}^{\rho}\left(s+\alpha_{i} T^{-1}\right)}{\partial x^{i}} \frac{\partial \tilde{x}^{q}(s)}{\partial \tau_{l}} \chi_{[0,+\infty)}(s)\right) d s,
\end{aligned}
$$

where $\chi_{[0,+\infty)}$ is as defined in (12). Substituting (33) into (31) yields

$$
\begin{aligned}
& \frac{\partial \tilde{g}_{k}^{q}(T, \sigma, \tau)}{\partial \tau_{l}}=\left(\frac{\partial \Phi_{k}\left(T, \tilde{x}^{q}(1)\right)}{\partial x}-w(1)^{\top}\right) \frac{\partial \tilde{x}^{q}(1)}{\partial \tau_{l}} \\
& +w\left(\tau_{l}\right)^{\top} \hat{f}^{l}\left(\tau_{l}\right)-w\left(\tau_{l}\right)^{\top} \hat{f}^{l+1}\left(\tau_{l}\right) \\
& \quad+\int_{0}^{1}\left\{\sum _ { \rho = 1 } ^ { q + 1 } \sum _ { i = 0 } ^ { m } \left(w\left(s+\alpha_{i} T^{-1}\right)^{\top} \frac{\partial \hat{f}^{\rho}\left(s+\alpha_{i} T^{-1}\right)}{\partial x^{i}}\right.\right. \\
& \left.\left.\quad \times \chi_{\mathcal{S}_{\rho}^{i}(T, \tau)}(s)\right)+\dot{w}(s)^{\top}\right\} \frac{\partial \tilde{x}^{q}(s)}{\partial \tau_{l}} d s .
\end{aligned}
$$

Choosing $w(\cdot)=\lambda_{k}^{q}(\cdot \mid T, \sigma, \tau)$ and applying (16)(18) completes the proof.

On the basis of Theorems 1-3, we now propose the following numerical procedure for computing the gradients of the cost and constraint functionals in Problem $(\mathrm{EP}(q))$.

Step 0. Input an integer $q \geq 1$ and a given triple $(T, \sigma, \tau) \in \mathcal{T} \times \Xi \times \Gamma$.

Step 1. Solve the system (13) from $s=0$ to $s=1$ to obtain $\tilde{x}^{q}(\cdot \mid T, \sigma, \tau)$.

Step 2. Using $\tilde{x}^{q}(\cdot \mid T, \sigma, \tau)$, compute $\tilde{g}_{k}^{q}(T, \sigma, \tau)$, $k \in\{0\} \cup \mathcal{E} \cup \mathcal{I}$.

Step 3. Using $\tilde{x}^{q}(\cdot \mid T, \sigma, \tau)$, solve the costate systems (16)-(18) from $s=1$ to $s=0$ to obtain $\lambda_{k}^{q}(\cdot \mid T, \sigma, \tau), k \in\{0\} \cup \mathcal{E} \cup \mathcal{I}$.

Step 4. Using $\tilde{x}^{q}(\cdot \mid T, \sigma, \tau)$ and $\lambda_{k}^{q}(\cdot \mid T, \sigma, \tau)$, compute $\partial \tilde{g}_{k}^{q}(T, \sigma, \tau) / \partial T, \partial \tilde{g}_{k}^{q}(T, \sigma, \tau) / \partial \sigma$, and $\partial \tilde{g}_{k}^{q}(T, \sigma, \tau) / \partial \tau$ via the formulae in Theorems $1-3$.

This procedure for calculating the gradients can be integrated with a standard nonlinear optimization method - e.g., sequential quadratic programming [14] - to solve Problem $(\operatorname{EP}(q))$ as a nonlinear programming problem.

\section{Numerical simulations}

To illustrate the solution procedure outlined in Section 3, we consider two versions of the renewable resource problem in [7]. To solve these problems, we wrote a Fortran program that combines the gradient computation procedure in Section 3 with the optimization software NLPQLP [15]. This program uses the 6 th order Runge-Kutta method to 
solve the state and costate systems. Lagrange interpolation [16] is used whenever the Runge-Kutta method requires the value of the state or costate at an intermediate time between two adjacent knot points.

\subsection{Version 1}

Consider the following model of a renewable resource as given in [7]:

$$
\begin{aligned}
& \dot{x}(t)=3 x(t)(1-0.2 x(t-0.5))-u(t), \\
& t \in[0, T], \\
& x(t)=2, \quad t \leq 0,
\end{aligned}
$$

where $x(t)$ is the biomass population and $u(t)$ is the harvesting effort. The cost functional is

$0.1 T^{2}+\int_{0}^{T} \exp (-0.05 t)\left(0.2 x(t)^{-1} u(t)^{3}-2 u(t)\right) d t$.

The following state constraint is imposed:

$$
x(t) \geq 2, \quad t \in[0, T] .
$$

Moreover, the control function and terminal time satisfy, respectively, the following bound constraints:

$$
u(t) \geq 0, \quad t \in[0, T],
$$

and

$$
T \in[0.6,20] \text {. }
$$

The problem is to minimize (36) subject to the dynamic system (34)-(35) and the constraints (37), (38) and (39). This problem is identical to the problem considered in [7].

Note that constraint (37) is a continuous state inequality constraint. Using the $\epsilon-\gamma$ approximation method [8], we approximate (37) by the following integral constraint:

$$
\gamma+\int_{0}^{T} \mathcal{L}_{\epsilon}(x(t)-2) d t \geq 0
$$

where $\epsilon>0$ and $\gamma>0$ are adjustable parameters and

$$
\mathcal{L}_{\epsilon}(\vartheta)= \begin{cases}\vartheta, & \text { if } \vartheta<-\epsilon, \\ -\frac{(\vartheta-\epsilon)^{2}}{4 \epsilon}, & \text { if }-\epsilon \leq \vartheta \leq \epsilon, \\ 0, & \text { if } \vartheta>\epsilon .\end{cases}
$$

Note that this integral constraint can be transformed into the canonical form (4) by introducing

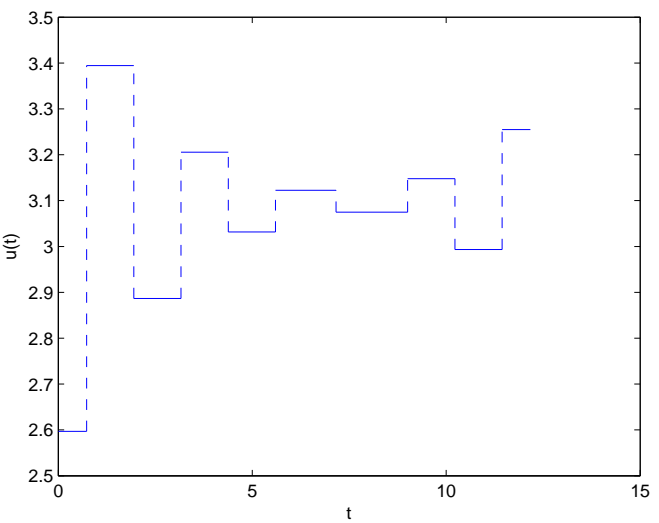

Figure 1: The optimal piecewise-constant control for Version 1.

an additional state variable; see Section 2 for details.

By running our program for $q=9$ and $\Delta=0.02$, we obtained an optimal terminal time of $T=$ 12.172181 and an optimal cost functional value of -26.068641 . These results are similar to the results obtained in [7]. The optimal piecewise-constant control is shown in Figure 1. Furthermore, the optimal state trajectory and the optimal costate trajectory are shown in Figures 2 and 3, respectively. In the computation process, we took $\epsilon=1.0 \times 10^{-2}$ and $\gamma=2.5 \times 10^{-3}$ as the initial values for the approximation parameters, adjusting them at each step according to the $\epsilon^{-} \gamma$ process. This process, which is described fully in [8] and the references cited therein, involves reducing $\gamma$ by a factor of 2 if the optimal solution satisfies (37), or reducing both $\epsilon$ and $\gamma$ by a factor of 10 if the solution does not satisfy (37). We terminated the $\epsilon-\gamma$ process when $\epsilon \leq 1.0 \times 10^{-8}$.

\subsection{Version 2}

Figure 2 shows that constraint (37) is never active at the optimal solution. This is consistent with the results in [7]. Thus, we modify the renewable resource problem by replacing (37) with the following continuous inequality constraint:

$$
x(t) \leq 4, \quad t \in[0, T] .
$$

Furthermore, we assume that the control function and terminal time satisfy, respectively, the following bound constraints:

$$
u(t) \in[0,3.5], \quad t \in[0, T],
$$




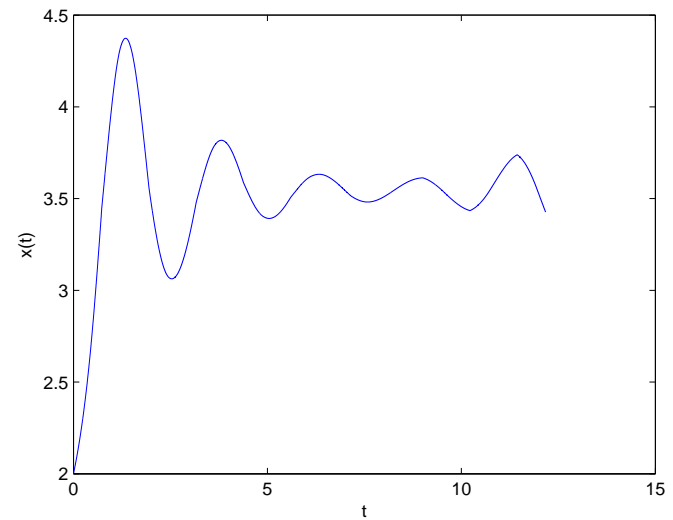

Figure 2: The optimal state trajectory for Version 1.

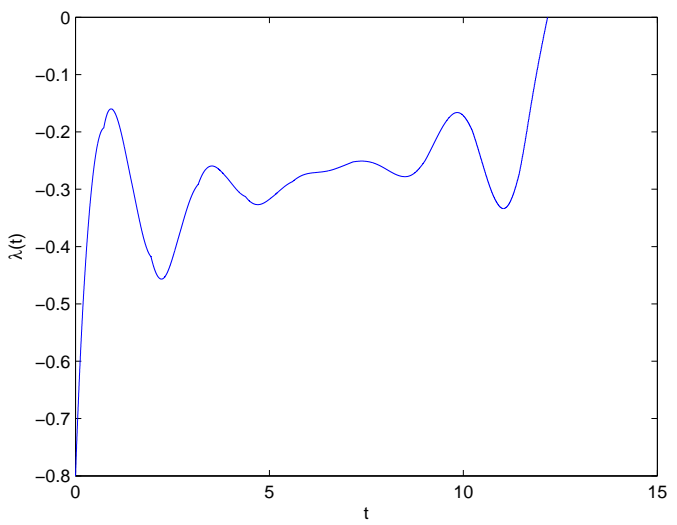

Figure 3: The optimal costate trajectory for Version 1.

and

$$
T \in[0.1,100] \text {. }
$$

Thus, our new optimal control problem is to choose the control function $u(t)$ such that the cost function (36) is minimized subject to the dynamic system (34)-(35) and the constraints (42), (43) and (44).

Using the $\epsilon^{-} \gamma$ approximation method [8], we approximate (42) by the following integral constraint:

$$
\gamma+\int_{0}^{T} \mathcal{L}_{\epsilon}(4-x(t)) d t \geq 0
$$

where $\epsilon>0$ and $\gamma>0$ are adjustable parameters, and $\mathcal{L}_{\epsilon}$ is as defined in (41).

By running the program for $q=9$ and $\Delta=0.02$, we obtained an optimal terminal time of $T=$ 12.15657 and an optimal cost functional value of

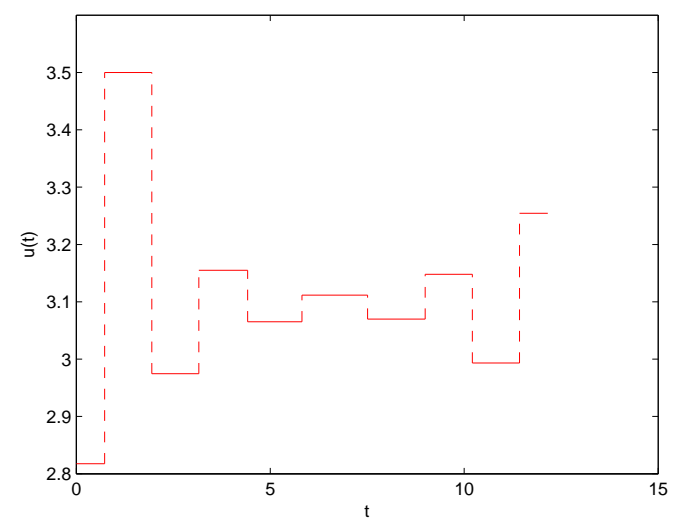

Figure 4: The optimal piecewise-constant control for Version 2 .

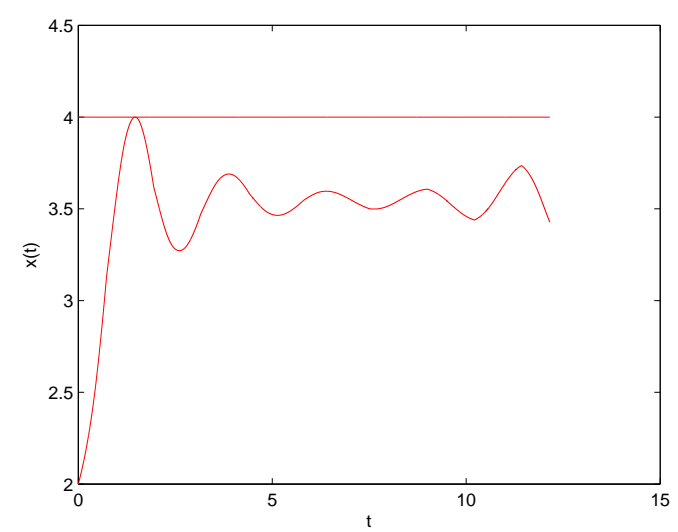

Figure 5: The optimal state trajectory for Version 2.

-25.972427. The optimal piecewise-constant control is shown in Figure 4. The optimal state trajectory is shown in Figure 5. In the computation process, we took $\epsilon=1.0 \times 10^{-2}$ and $\gamma=2.5 \times 10^{-3}$ as the initial values for the approximation parameters, adjusting them at each step according to the same $\epsilon^{-} \gamma$ process as in Version 1. From Figure 5, we immediately see that the continuous inequality constraint (42) is always satisfied, and is active at one point.

\section{Conclusion}

In this paper, the time-delay optimal control problem with free terminal time was investigated. Using the well-known time-scaling transformation, 
we transformed this optimal control problem into an equivalent problem with fixed terminal time and variable time-delays. We then applied the control parameterization method to yield a finitedimensional approximate problem, for which a gradient-based optimization algorithm was proposed. Numerical results show that this algorithm is highly effective at solving time-delay optimal control problems with free terminal time. It is worth noting that the piecewise-constant control approximation method used in this paper can be extended to piecewise-linear or piecewise-quadratic approximation schemes if a continuous control is required (see [8]).

\section{Acknowledgements}

The first author is supported by the Natural Science Foundation for the Youth of China (Grant No. 11201267). The second author is supported by the Natural Science Foundation of China (Grant No. 11350110208) and the Australian Research Council (Discovery Grants DP110100083, DP140100289). The third author is supported by the Australian Research Council (Discovery Grants DP110100083, DP140100289).

\section{References}

[1] J.D.L. Rowland, R.B. Vinter, A maximum principle for free endtime optimal control problems with data discontinuous in time, IEEE Trans. Automat. Control 14 (1976) 613-622.

[2] H. Maurer, H.J. Oberle, Second order sufficient conditions for optimal control problems with free final time: The Riccati approach, SIAM J. Control Optim. 41 (2002) 380-403.

[3] K.L. Teo, C.J. Goh, C.C. Lim, A computational method for a class of dynamical optimization problems in which the terminal time is conditionally free, IMA J. Math. Control Inform. 6 (1989) 81-95.

[4] Q. Lin, R. Loxton, K.L. Teo, Y.H. Wu, A new computational method for a class of free terminal time optimal control problems, Pac. J. Optim. 7 (2011) 63-81.

[5] Q. Lin, R. Loxton, K.L. Teo, Y.H. Wu, Optimal control computation for nonlinear systems with statedependent stopping criteria, Automatica J. IFAC 48 (2013) 2116-2129.

[6] H. Maurer, N.P. Osmolovskii, Second order sufficient conditions for time-optimal bang-bang control, SIAM J. Control Optim. 42 (2004) 2239-2263.

[7] A. Boccia, P. Falugi, H. Maurer, R.B. Vinter, Free time optimal control problems with time delays, Proceedings of the 52nd IEEE Conference on Decision and Control, Florence, Italy, 2013, pp. 520-525.

[8] Q. Lin, R. Loxton, K.L. Teo, The control parameterization method for nonlinear optimal control: A survey, J. Ind. Manag. Optim. 10 (2014) 275-309.
[9] L. Göllmann, D. Kern, H. Maurer, Optimal control problems with delays in state and control variables subject to mixed control-state constraints, Optimal Control Appl. Methods 30 (2014) 341-365.

[10] N.U. Ahmed, Elements of Finite-dimensional Systems and Control Theory, Longman Scientific and Technical, Essex, 1988.

[11] R. Loxton, K.L. Teo, V. Rehbock, W.K. Ling, Optimal switching instants for a switched-capacitor DC/DC power converter, Automatica J. IFAC 45 (2009) 973980.

[12] Q. Lin, R. Loxton, K.L. Teo, Y.H. Wu, A new computational method for optimizing nonlinear impulsive systems, Dyn. Contin. Discrete Impuls. Syst. Ser. B Appl. Algorithms 18 (2011) 59-76.

[13] H. Maurer, C.Büskens, J.H.R. Kim, C.Y. Kaya, Optimization methods for the verification of second order sufficient conditions for bang-bang controls, Optimal Control Appl. Methods 26 (2005) 129-156.

[14] J. Nocedal, S.J. Wright, Numerical Optimization, Springer-Verlag, New York, 1999.

[15] K. Schittkowski, NLPQLP: A Fortran implementation of a sequential quadratic programming algorithm with distributed and non-monotone line search - User's guide, University of Bayreuth, Bayreuth (2007)

[16] J. Stoer, R. Bulirsch, Introduction to Numerical Analysis, Springer-Verlag, New York, 1980 